\title{
Liquid Crystals Phase Transitions and AC-Calorimetry
}

\author{
Krishna P. Sigdel \\ Dept of Physics, Worcester Polytechnic Institute, Worcester, Massachusetts 01609, USA \\ $<$ kps@wpi.edu>
}

\begin{abstract}
Liquid crystal is a delicate and beautiful phase of matter showing the order in between liquid and crystals. They have different phases and phase transitions. A powerful tool called AC calorimetry can be used to characterize the different phases and phase transitions. In this article, use of ac-calorimetry technique in liquid crystal phases and phase transitions is described.
\end{abstract}

\section{Introduction}

Liquid crystal (LC) $[1,2]$ is an anisotropic fluid which exhibits both the properties of solid and liquid. Its name suggests that it is an intermediate phase of matter in between the liquid and crystal. The molecules in a crystal (solid) generally possess both positional and orientation order while the molecules in a liquid do not have any order and can move freely in random manner. The liquid crystals molecules possess orientation and partial translational order. Liquid crystals can flow as a liquid and at the same time its molecules may be oriented in a certain direction like the molecules in a crystal do. LC possesses some crystalline properties such as magnetic, electric and optical anisotropy, periodic arrangement of molecules in one spatial direction as well as some typical properties of a liquid such as fluidity, elasticity and formation of droplets. Typically the molecules are rod-like or disklike. The rod like molecules or rod-like mesogens is elongated $\left(\approx 25^{\circ} \mathrm{A}\right)$ and has anisotropic geometry which leads to preferential alignment along one spatial direction. Disc-like molecules or discotic mesogens are flat and have more or less disc-shaped central core leading to two dimensional columnar ordering. A large number of well-known compounds are liquid crystals e.g. cellulose, DNA, cholesterol esters, lecithin, paraffin.

The credit for the discovery of liquid crystal goes to an Austrian botanical physiologist Friedrich Reinitzer, even though the liquid crystalline phase was observed in the past as well. In 1888 Reitnizer observed two melting temperatures of cholesteryl benzoate. At $145.5^{\circ}$ it melts forming a cloudy liquid that become clear at $178.5^{\circ}$. Reitnizer was puzzled with this peculiar feature shown by the compound and wrote to physicist Otto Lehmann seeking help from later's expertise of polarizing microscopy. After the observation of the sample gotten from Reinitzer , Lehmann realized that the cloudy liquid observed was a new state of matter and coined name "liquid crystal", illustrating that it is something between a liquid and a solid, sharing important properties ofboth.

Liquid crystal technology has had a major effect in many areas of science and technology $[3,4]$. The most common application of liquid crystal technology is liquid crystal displays which have grown to a multi-billion companies. From simple wrist watch, computer to an advanced computer screen LCDs have evolved into a versatile interface. LCD uses much less power than that CRTs use.

LCs can be thermotropic, lyotropic or amphotropic. Thermotropic LCs are induced by thermal process. They show phase behavior change as a function of temperature. Thermotropic mesogens can be obtained either lowering the temperature of its isotropic phase or raising the temperature of its solid phase. Lyotropic LCs are induced by solvent. Their phase behavior change as a function of concentration of solvent. Amphotropic LCs show both thermotropic and lyotropic behavior. LC displays varieties of intermediate thermody-namically stable phases but the most common and important are "Nematic" and "Smectic". In nematic phases (Fig. 1) the molecules are free to move in all directions i.e. there is no positional order of centers of mass but they tend to orient in a certain direction. 


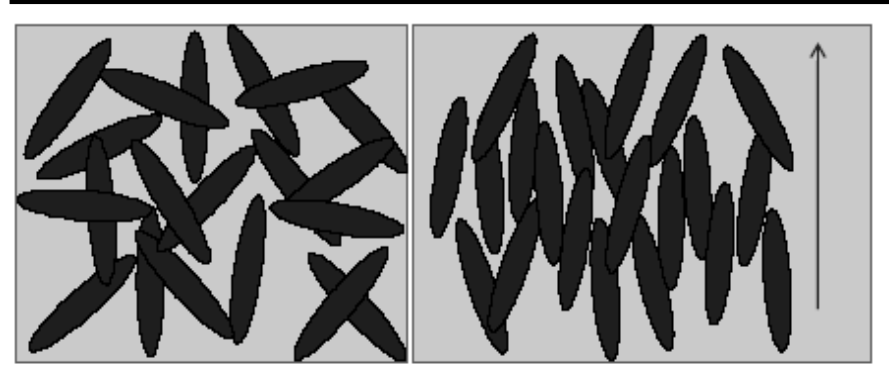

Isotropic

Nematic

\section{FIG. 1: Cartoons of nematic and isotropic phases}

The direction of preferred orientation can be described by a unit vector, $\hat{n}$, is called nematicdirector. Smectic phases (Fig. 2) have a layered structure with the molecules oriented parallel or tilted to the layer normal. In smectic-A, molecules are parallel to layer normal and in smectic-C phase the molecules are tilted with respect to layer normal. They are characterized by absence of positional order within the layers i.e. molecules have some freedom to move within the layer but they are much free to move between the layers. Smectics can be considered as stacks of two dimensional fluids but they behave as crystalline across the layers.

The orientational order can be described by a symmetric and traceless second rank tensor

(Qij) [2] and is established in three dimensions. Nematic order can be described by a scalar parameter $S$ which measures the magnitude of orientational order about the orientational axis on short length scale and on longer length scale by a vector, $\hat{n}$ called nematic director, which describes spatial orientation of this axis. These measure of nematic order are related to nematic order parameter by $Q_{i j}=\frac{1}{2} S\left(3 \hat{n}_{i} \hat{n}_{j}-\delta_{i j}\right)$. The Smectic-A order parameter $\psi$ is defined as the amplitude of a one

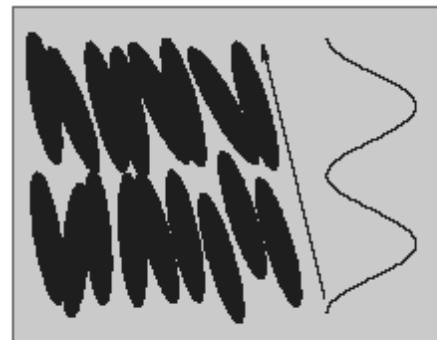

Smetic- C

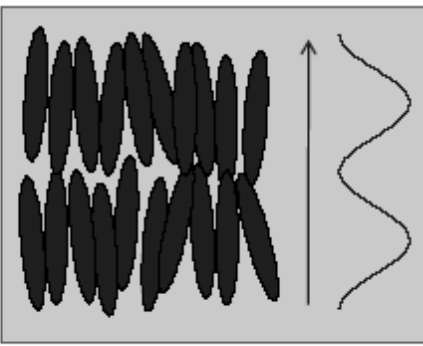

Smetic- A
FIG. 2: Cartoons of Smectic-A and Smectic-C Phases dimensional wave and is related to molecular density as $\rho(\vec{r})=\operatorname{Re}\left[\rho_{0}+\exp \left(i \overrightarrow{q_{0}} \cdot \vec{r}\right) \psi(\vec{r})\right]$, where $q_{0}=2 \pi / d$ is the wave vector corresponding to the layer spacing $d$ and complex field $\psi(\vec{r})=\psi_{0} \exp \left(i \overrightarrow{q_{0}} \cdot \vec{r}\right)$.

There are many successful methods to study the Liquid Crystals at different phase transitions; some of them include calorimetry, x-ray diffraction, neutron scattering, light scattering, NMR, optical microscopy, etc. Among the different calorimetric techniques to study liquid crystals, traditional adiabatic calorimetry has high precision and can be used to determine the latent heat at strongly firstorder transitions. However, this method does not have enough resolution possibilities to characterize a second-order transition and requires a large sample of several grams to get reliable data [5]. Differential scanning calorimetry has high sensivity, making it a widely used method because of its ease of operation and small sample size $(10 \mathrm{mg})$. Due to rapid scan rates of $1-5 \mathrm{~K} / \mathrm{min}$, the precision and absolute accuracy in enthalpy changes is modest, and the temperature resolution is poor [6]. Since its introduction into the literature during the late 1960s [7], the AC calorimetry has developed into a very powerful, well-established and widely used technique for studying a variety of phase transitions in liquid crystals, polymers and biological systems.

In this article we explain briefly ac-calorimetric technique to study the effect of nonmesogenic, low molecular weight, solvent(hexane) concentration on the isotropic to nematic $(I-N)$ and nematic to smectic$\mathrm{A}(N-\operatorname{Sm} A)$ phase transitions on octylcyanobiphenyl and hex- ane $(8 \mathrm{CB}+$ hex $)$ binary mixtures as the function of hexane concentrations. Our present work is organized as follows: Section II describes the preparation of sample and the cell as well as the experimental ac-calorimetric procedure which we employed to this work. Section III describes the result of our study of $8 \mathrm{CB}+$ hex system. Finally, the section IV summaries the conclusion drawn.

\section{Experimental Procedure}

\section{A. Operation}

AC-calorimetry, originally introduced by Sullivan 
and Siedel [7], is a semi classical, well established, widely used and an extremely valuable tool in the study of phase transitions. In ac-technique, heat capacity is measured in quasi-equilibrium condition which is very crucial because of the fact that most of the phase transition theories are based on the equilibrium condition considerations. AC-calorimetric technique has several advantages as compared to the other calorimetric methods. We can measure the heat capacity of very small amount of sample ranging from $g$ to $m g$ in very high temperature range with high temperature resolutions. It can be extended to get the information about the characteristics like thermal conductivity and dynamic heat capacity $C p(!)$. Ac technique is a versatile and easy to use.

It can be used to study the low-temperature properties of superfluid helium films adsorbed in porous glasses [8], superconducting films[9]; studies on melting of nitrogen on graphite [10]; and studies of phase transitions in bulk liquid crystals [11], and in free standing liquid crystal films [12] as a few examples.

A simple model to describe this technique is called one-lump thermal model (Fig.3). This consists of a cell containing a sample with a heater and thermometer attached to its surface. The $\mathrm{Ti}, \mathrm{Ci}$, and $R i$ are the temperature, heat capacity, and the thermal resistance of the sample, thermometer, heater, and bath respectively, labelled by the indices $i=S, \theta, H$, and $B$.

In the ac-mode of an ac-calorimetry, oscillating heating power $P_{a c} \exp (i \omega t)$ is applied to the cell resulting in temperature oscillations with amplitude $T a c$ and a relative phase shift between $T a c$ and input power, $\varphi=\Phi+\pi / 2$, where $\Phi$ is the absolute phase shift between Tac and the input power. The relative phase shift also provides crucial information regarding the order of the phase transition [13]. With the definition of the heat capacity amplitude,

\section{Cell}

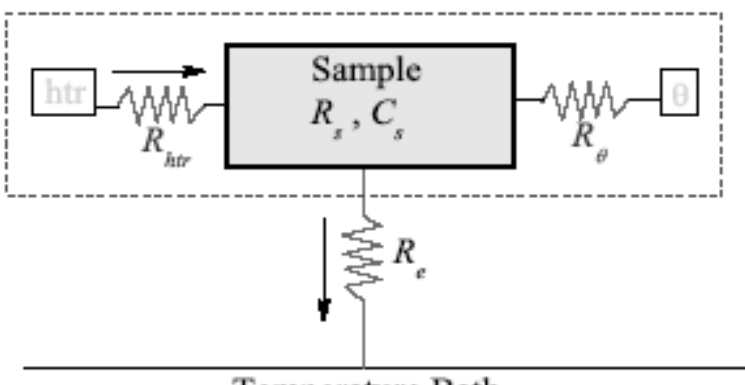

Temperature Bath

FIG. 3: One-lump thermal model used in accalorimetry

$C^{*}=P_{a c} /\left(\omega T_{a c}\right)$, the specific heat at a heating frequency $\omega$ can be expressed as

$$
\begin{gathered}
C_{p}=\frac{C_{\text {filled }}^{\prime}-C_{\text {empty }}}{m_{s}}=\frac{C^{*} \cos (\varphi) f(\omega)-C_{\text {empty }}}{m_{s}} \\
C^{\prime \prime}=C_{\text {filled }}^{\prime \prime}=C^{*} \sin (\varphi) g(\omega)-\frac{1}{\omega R_{e}}
\end{gathered}
$$

where $C_{\text {filled }}^{\prime}$ and $C^{\prime}{ }_{\text {filled }}$ are the real and imaginary parts of the heat capacity which represent the storage (capacitance) and loss (dispersion) of the energy in the sample respectively, $C_{\text {empty }}$ is the heat capacity of the empty cell, $m_{s}$ is the mass of the sample (in the range of $15 \mathrm{mg}$ to $40 \mathrm{mg}$ ), and $R_{e}$ is the external thermal resistance between the cell and the bath. The functions $f(\omega) \approx g(\omega) \approx 1$ are small correction factors due to the non-negligible internal resistance $R_{i}$ of the sample compared to $R_{e}[14]$.

The excess specific heat associated with a phase transition can be determined by subtracting an appropriate background $C_{P}^{B ;}$ from the total specific heat over a wide temperature range; $\Delta C_{p}=C_{p}-C_{P}^{B G}$ . Fig. 5(a) illustrates this subtraction; the dash-dot line represents the background.

The pretransitional enthalpy associated with a phase transition is defined as

$$
\delta H=\int \Delta C_{p} d T
$$

where the integration is usually carried over as wide a temperature range as possible. The integration of the imaginary part of heat capacity given by Eq. (2) yields the imaginary transition enthalpy $\pm H^{\prime}$, which 
is the dispersion of energy in the sample and a proxy of latent heat associated to the phase transition. Fig. 5(a) and (b) illustrates the way how we calculate the effective ac-enthalpy change $\delta H_{I N}^{*}, \delta H_{N A}^{*}$ and imaginary enthalpy change $\delta H_{I N}^{n}$ associated with phase transitions. In ac-calorimetric technique the uncertainty in determining the enthalpy is typically $10 \%$ due to the base line and background subtractions.

\section{B. Sample preparation and cell design}

The liquid crystal $8 \mathrm{CB}$, purchased from Frinton Laboratory, has the chemical formula $\mathrm{C}_{8} \mathrm{H}_{17}$ $\mathrm{C}_{6} \mathrm{H}_{5}-\mathrm{C}_{6} \mathrm{H}_{5}-\mathrm{CN}$, a molecular mass $M_{w}=291.44$ $\mathrm{g} \mathrm{mol}^{-1}$, and a density of $\rho_{\mathrm{LC}}=0.996 \mathrm{~g} \mathrm{ml}^{-1}$. Pure $8 \mathrm{CB}$ has a weakly first-order, isotropic to nematic

phase transition at $T_{N}^{0}=313.98 \mathrm{~K}$, a second order nematic to smectic- $A$ transition at $T 0 N A=306.97$ $\mathrm{K}$, and a strongly first-order crystal to $\operatorname{Sm} A$ transition at $T 0 C r A=290 \mathrm{~K}$ [15]. The single batch of $8 \mathrm{CB}$ used for pure and mixture samples was degassed under vacuum for about two hours in the isotropic phase before use. Spectroscopic grade (ultralow water content with a nominal $99.9 \%$ purity) n-hexane purchased from EM Science was used without further purification. The pure n-hexane has molecular formula $\mathrm{C}_{6} \mathrm{H}_{14}$, molecular mass of 86.18 $\mathrm{g} \mathrm{mol}^{-1}$, a density of $0.6548 \mathrm{~g} \mathrm{ml}^{-1}$, and a boiling point of $342 \mathrm{~K}$ with no known mesogenic phases. The $8 \mathrm{CB}$ and $\mathrm{n}$-hexane mixtures appear by visual inspection to be miscible up to an n-hexane mole fraction of 0.119 . Measurements were performed on samples as a function of n-hexane mole fraction, $\mathrm{x}_{\text {hex }}=n_{\text {hex }} /\left(n_{\text {hex }}+n_{L C}\right)$, where $n_{\text {hex }}$ and $n_{L C}$

where $n_{h e x}$ and $n_{L C}$ are the moles of hexane and liquid crystal used ranging from 0 (pure $8 \mathrm{CB}$ ) to 0.12 . All $8 \mathrm{CB}+$ hex samples experienced the same thermal history with data taken at a heating frequency of $0.03125 \mathrm{~Hz}$ and a final slow scanning rate of $\pm 0.4 \mathrm{Kh}^{-1}$.

An aluminum "envelope" heat capacity cell, 15 $\mathrm{mm}$ in length, $8 \mathrm{~mm}$ in width, and $\approx 0.5 \mathrm{~mm}$ thick was prepared from a sheet of aluminum which was cleaned in progressive applications of water, ethanol and acetone in an ultrasonic bath. The sheet was then folded and sealed on three sides with super-glue
(Cyanoacrylate). Once the cell was thoroughly dried, the desired amount of liquid crystal followed by a relatively large amount of n-hexane was introduced to the cell. The mass of the sample and cell was monitored as the n-hexane was allowed to evaporate slowly until the desired mass of the n-hexane was achieved. At the point of the desired mass of the $8 \mathrm{CB}+$ hex mixture, the envelop ${ }^{\circ}$ ap was quickly folded and sealed with the super-glue. Careful massing of super-glue and sample during every stage of this process ensured the proper amounts of materials were sealed within the cell. Extensive care was taken in handling the sample due to relatively rapid evaporative nature of $n$-hexane and total mass of the cell was periodically checked. The sample cell consists of an aluminium envelop mentioned above and is closely matching the size of the heater. After the sample was introduced into the cell a $120-\Omega$ strain gauge heater and $1-M \Omega$ carbon-flake thermistor were attached on its surfaces. The filled cell was then mounted in the high resolution calorimeter,the details of which can be found elsewhere $[7,13,16]$.

High resolution ac-calorimetric measurements were carried out at WPI the block diagram of which is shown in Fig. 4. It consists of a copper block the temperature of which is controlled by a Lakeshore temperature controller via a platinum resistance thermometer (PRT) and a flexible heater. A thermistor and a heater are attached to the opposite faces of the sample-cell and are connected to Keithley digital multimeter and HP function generator respectively. HP function generator provides the oscillating power to the cell via heater and the multimeter measures the temperatures responses via the thermistor as well as center PRT.

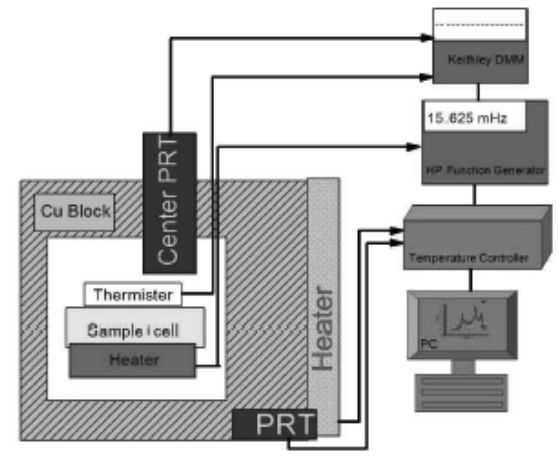

FIG. 4: Block diagram of an AC-Calorimeter. 
The Himalavan Phvsics. Vol.1. No.1. Mav 2010

\begin{tabular}{ccccccc}
\hline \hline $\mathrm{x}_{\text {hex }}$ & $T_{I N}$ & $T_{N A}$ & $\Delta T_{\text {nem }}$ & $\delta H_{I N}^{*}$ & $\delta H_{N A}^{*}$ & $\delta H_{I N}^{\prime}$ \\
\hline 0.00 & $313.20 \pm 0.07$ & 306.10 & $7.11 \pm 0.11$ & $4.67 \pm 0.47$ & 0.49 & $0.59 \pm 0.06$ \\
0.02 & $309.95 \pm 0.39$ & 304.41 & $5.53 \pm 0.59$ & $3.99 \pm 0.40$ & 0.83 & $0.33 \pm 0.03$ \\
0.03 & $309.12 \pm 0.27$ & 304.16 & $5.03 \pm 0.41$ & $4.19 \pm 0.42$ & 0.98 & $0.40 \pm 0.04$ \\
0.06 & $309.48 \pm 0.33$ & 304.20 & $5.27 \pm 0.50$ & $3.36 \pm 0.34$ & 0.52 & $0.32 \pm 0.03$ \\
0.08 & $308.06 \pm 0.42$ & 303.91 & $4.46 \pm 0.63$ & $3.79 \pm 0.38$ & 1.08 & $1.27 \pm 0.13$ \\
0.09 & $305.16 \pm 0.64$ & 302.10 & $3.23 \pm 0.96$ & $4.11 \pm 0.41$ & 1.01 & $0.97 \pm 0.10$ \\
0.12 & $303.62 \pm 0.94$ & 301.45 & $2.53 \pm 1.41$ & $4.09 \pm 0.41$ & 0.73 & $1.15 \pm 0.12$ \\
\hline \hline
\end{tabular}

TABLE I: Summary of the calorimetric results for pure $8 \mathrm{CB}$ and the $8 \mathrm{CB}+$ hex samples on heating. Shown are $\mathrm{n}$-hexane molar fraction $\mathrm{x}_{h e x}, I-N$ transition temperature $T_{I N}, N-\operatorname{Sm} A$ transition temperature $T_{N A}$, nematic range $\Delta$ Them (in Kelvins), effective ac-enthalpy change $\delta H_{I N}^{*}, \delta H_{N A}^{*}$ and dispersive enthalpy $\delta H_{I N}^{*}$ (in $\mathrm{J} / \mathrm{g}$ ).

\section{Results and Discussions}

The summary of our result for $8 \mathrm{CB}$ +hex samples including pure $8 \mathrm{CB}$ is tabulated in table

I. Included are the $I-N$ and $N-\operatorname{Sm} A$ transition temperatures and nematic range measured in Kelvins, $\delta H_{I N}^{*}, \delta H_{I N}^{*}$, and $\delta H_{N A}^{*}$ measured in $\mathrm{J} / \mathrm{g}$.

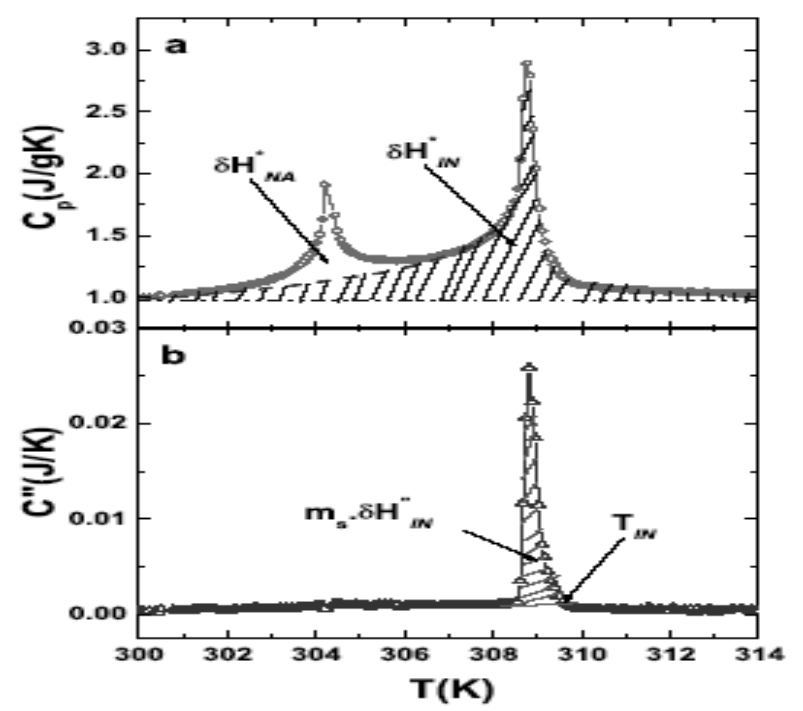

FIG. 5: (a) The specific heat on heating as a function of temperature for the xhex $=0.06$ sample illustrating the overall background (dashed-dot) and low-temperature wing under the N-SmA peak (dashed) behavior used to determine $\triangle C p, \delta H^{*} I N$ and $\delta H^{*} N A$. b) Dispersive part of heat capacity on heating for the xhex $=0.06$ sample illustrating $\delta H$ 'IN and the I-N transition temperature $T_{I N}$.

Fig. 5 shows the background heat capacities and illustrates how we calculated the enthalpies and transition temperatures. Fig. 6 shows the typical heat capacity and phase difference between applied power and temperature oscillation profile as a function of temperature for pure $8 \mathrm{CB}$ obtained by accalorimetry. $\mathrm{C}^{*}$ and $\varphi$ shows discontinuity at phase transitions. Two peaks in $C^{*}$ represent phase transitions-low-temperature showing $N-\operatorname{Sm} A$ and high-temperature one showing $I-N$ phase transition. $\varphi$ gives the information about the order of phase transitions. In pure $8 \mathrm{CB} I-N$ transition is first order (where latent heat presents) and $N-\operatorname{Sm} A$ transition is of second order (no latent heat, occurred due to energy fluctuation only).

The resulting excess specific heat data for $8 \mathrm{CB}+$ hex samples on heating are shown in Fig. 7(a) as a function of temperature. Here $\delta C p$ are presented in $J K^{-1}$ per gram of the sample. The $\delta C p$ peaks of both the $I-N$ and $N-A$ transitions shift towards lower temperature. $I-N \delta C p$ peaks are progressively smearing with increasing $\mathrm{x}_{h e x}$ whereas $N-A$ peaks are narrowing and becoming larger in height. Fig. 7(b) shows the dispersive part of heat capacity $C$ " for pure $8 \mathrm{CB}$ and $8 \mathrm{CB}+$ hex samples on heating. The $I-N$ transition $C$ " peaks are also smeared with progressively longer tail on the isotropic side as the hexane concentration increases. C" $N-A$ peaks are observed only for xhex $=0.08 ; 0.09 ; 0.12$. This means latent heat is present only for these hexane mole fraction and are ${ }^{-}$rst order phase transition whereas for lower concentration 
Sigdel/Liquid...

$N-A$ transition dos not have any latent heat and of second order. Therefore there is a cross over between ${ }^{-}$rst order and second order $N-A$ transition. This crossover is call tricritical point which is about $\mathrm{x}$ hex $=0.07$ for $8 \mathrm{CB}+$ hex system. Interestingly, the temperature of the C" peak does not coincide with the temperature of the $\Delta C p$ peak on heating. Because of the mismatch between $C$ " and $\Delta C p$ peaks in

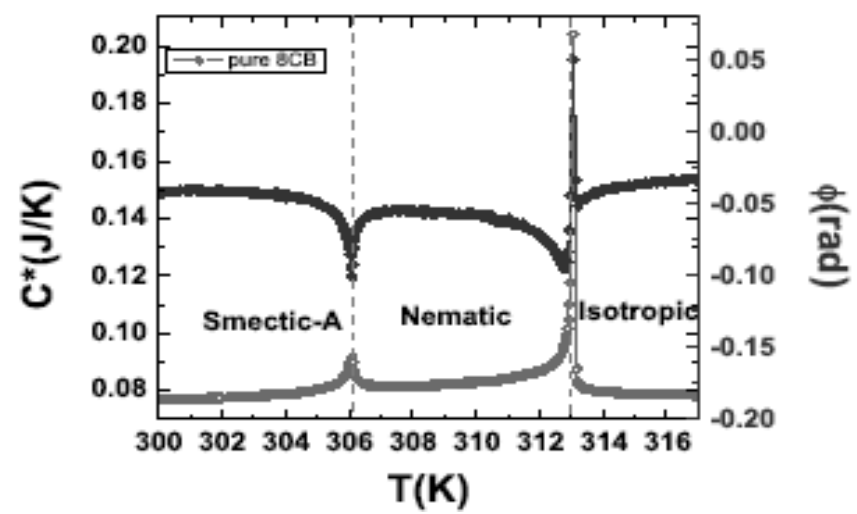

FIG. 6: Heat capacity (left axis) and phase di $\AA_{\text {erence }}$ between the applied power and temperature oscillation (right axis) as a function of temperature for pure $8 C B$ on heating.

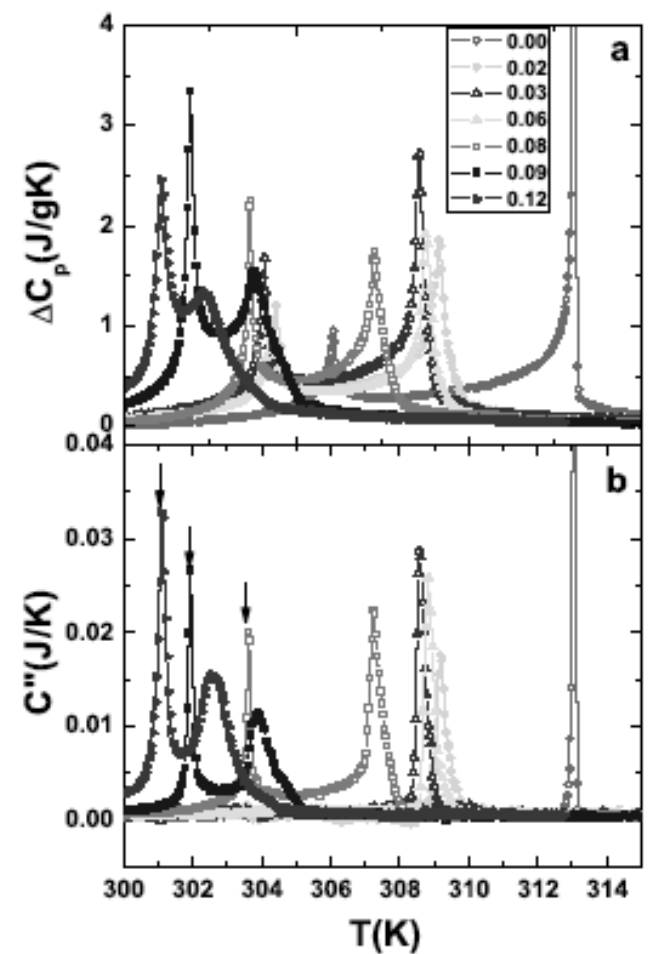

FIG. 7: (a) The excess specific heat $\delta C p$ associated with the I-N and $N$-A transition on heating as a function of temperature for pure and all $8 C B+$ hex

samples. The definition of the symbols is given on the inset. (b)The imaginary part of heat capacity on heating for all samples as a function of temperature.

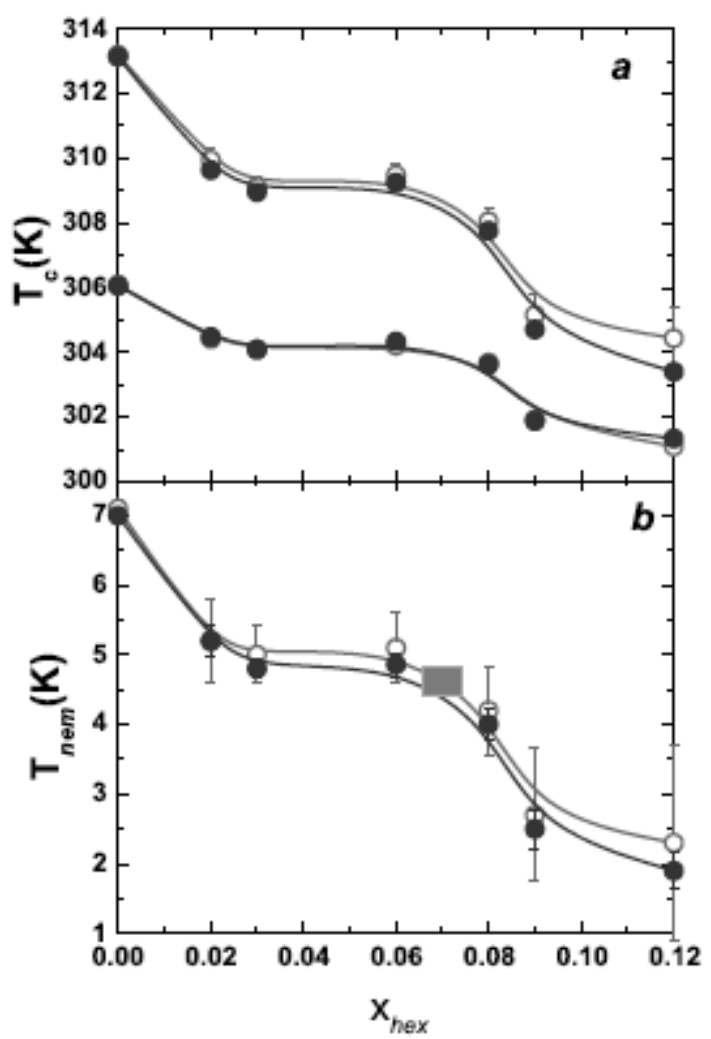

FIG. 8: (a) The I-N and N-SmA phase transition temperatures on heating (o) and cooling (•) as a function of xhex. Solid lines are guides to the eye. (b) The nematic temperature range $\phi$ Tnm on heating (o) and cooling $(\bullet)$ as a function of $x$ hex. The rectangular box represents the position of tricritical point for $8 C B+$ hex system.

temperature and long $\Delta C p$ tail on the isotropic side, the temperature of the $I-N$ transition on heating is taken as the approximate inflection point of C" on the isotropic side. The $I-N$ and $N-\operatorname{Sm} A$ phase transition temperatures as a function of n-hexane mole fraction are shown in Fig. 8(a) for heating and cooling scans. The $I-N$ phase transition temperature $T_{I N}$ is defined as the high temperature limit of the $I+N$ co-existence region from isotropic to nematic region, determined from C" for heating and cooling scans. See Fig. 5(b) which illustrates this procedure. The $N$-Sm $A$ phase transition temperature $T_{N A}$ was 
determined in two ways; for lower xhex samples it is simply the $N-\operatorname{Sm} A \Delta C p$ peak temperature, whereas for higher concentrations, where the $N-\operatorname{Sm} A$ transition exhibits a peak in $C^{\prime \prime}, T_{N A}$ is taken as high temperature limit of $N-\operatorname{Sm} A C$ " peak. The $I-N$ transition temperatures for heating and cooling as function of $\mathrm{xhex}$ are consistent with each other, but $T_{I N}$ on cooling becomes progressively lower than on heating with increasing xhex. As the mole fraction of n-hexane increases the transition temperatures decrease nonlinearly with a plateau or bump at xhex $\approx 0.07$ for both $T_{I N}$ and $T_{N A}$. See Figure 8(a). Figure 8 (b) shows the nematic temperature range $\Delta T n m$ $=T_{I N}-T_{N A}$ as a function of $\mathrm{n}$-hexane mole fraction revealing an overall decrease in $\Delta T n m$ with a plateau similar to that seen in $T_{I N}(\mathrm{x} h e x)$ and $T_{N A}(\mathrm{xhex})$. The nematic range decreases from $7.11 \mathrm{~K}$ for pure $8 \mathrm{CB}$ to $2.53 \mathrm{~K}$ for the $\mathrm{xhex}=0.12$ sample. The behavior of $\Delta T n m$ simply indicates the greater suppression of orientational order (nematic phase) due to the presence of the solvent.

\section{Conclusion}

We have undertaken a calorimetric study on the effect of non- mesogenic, low molecular weight solvent (hexane) on octylcyanobiphenyl (8CB) on the $I-N$ and $N-\operatorname{Sm} A$ phase transitions. The both the transition temperatures shifts to lower temperatures with increasing the hexane concentration on the binary mixture. But the effect of the hexane for depressing the $N-\operatorname{SmA}$ transition is smaller as compared to that of $I-N$ transition temperature. The result obtained reveals new aspect of the effect of nonmesogenic disorder on the liquid crystal transition. It is concluded that as the concentration of hexane increases the coupling between order parameters and the molar fraction $(\mathrm{x})$ increases indicating the decrease in nematic range. The dilution of the liquid crystal causes the decrease in liquid crystal molecule interaction which consequently gives rise the evolution of the phase transitions and cross over between second order to first order $N-\operatorname{Sm} A$ transition.

\section{References:}

[1] S. Chandrashekhar, Liquid Crystals (Cambridge University Press, England, 1992).

[2] P. G. de Gennes and J. Prost, The Physics of Liquid Crystals (Oxford University Press, Clarendon, Oxford, England, 1993).

[3] B. B. eds., Liquid Crystals-Applications and Uses (1-3 World Scientific, Singapore, 1990).

[4] D. Demus, J. Goodby, G. Gray, H. Spiess, and V. V. eds., Hand Book of Liquid Crystals (1 Wiley-VCH,Weinheim, Canada, 1998).

[5] M. Anisimov, Critical Phenomena in Liquids and Liquid Crystals (Gordon and Breach, 1991).

[6] J. Thoen, Int. J. Mod. Phys. B 9, 2157 (1995).

[7] P. F. Sullivan and G. Seidel, Phys. Rev. 173, 679 (1968).

[8] Y. P. Feng, A. Jin, D. Finotello, K. Gillis, and M. Chan, Phys. Rev. B 38, 7041 (1988).

[9] T. Kenny and P. L. Richard, Rev. Sci. Instrum. 61, 822 (1990).

[10] B. Zhou, G. S. Iannacchione, and C. W. Garland, Phys. Rev. Lett. 56, 1579 (1986).

[11] J. Thoen, H. Marynissen, and G. M. Mochel, Phys. Rev. A 26, 2886 (1982).

[12] R. Geer, T. Stoebe, T. Pithford, and C. C. Haung, Rev. Sci. Instrum. 62, 415 (1991).

[13] D. Finotello, S. Qian, and G. S. Iannacchione, Thermochimica Acta 304/305, 303 (1997).

[14] A. Roshi, G. S. Iannacchione, P. S. Clegg, and R. J. Birgeneau, Phys. Rev. E 69, 031703 (2004).

[15] G. S. Iannacchione, C. W. Garland, J. T. Mang, and T. P. Rieker, Phys. Rev. E 58, 5966 (1998).

[16] H. Yao and C. W. Garland, Rev. Sci. Instrum. 69, 172 (1998).

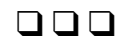

